Published in final edited form as:

Endocrine. 2017 December ; 58(3): 413-425. doi:10.1007/s12020-017-1435-x.

\title{
Estrogen-Based Hormone Therapy in Women with Primary Ovarian Insufficiency: A Systematic Review
}

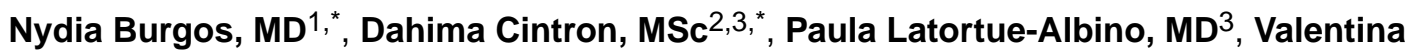 \\ Serrano, MD ${ }^{4,5}$, Rene Rodriguez Gutierrez, MD, MSc ${ }^{4,6,7,8}$, Stephanie Faubion, MD $^{9}$, \\ Gabriela Spencer-Bonilla, BA ${ }^{2,4}$, Patricia J Erwin, MLS $^{10}$, and Mohammad Hassan Murad, \\ MD, MPH ${ }^{11}$ \\ ${ }^{1}$ Internal Medicine Department, VA Caribbean Health Care System, San Juan, 00921, PR \\ ${ }^{2}$ Mayo Graduate School, Mayo Clinic, Rochester, MN, 55905, USA \\ ${ }^{3}$ University of Puerto Rico-Medical Sciences Campus, San Juan, 00921, PR \\ ${ }^{4}$ Knowledge and Evaluation Research Unit in Endocrinology, Division of Endocrinology, Diabetes, \\ Metabolism and Nutrition, Department of Medicine, Mayo Clinic, Rochester, MN, 55905, USA \\ ${ }^{5}$ Department of Nutrition, Diabetes and Metabolism, Escuela de Medicina, Pontifica Universidad \\ Católica de Chile. Santiago. Chile \\ ${ }^{6}$ Division of Endocrinology, Diabetes, Metabolism and Nutrition, Department of Medicine, Mayo \\ Clinic, Rochester, MN, 55905, USA \\ ${ }^{7}$ Division of Endocrinology, Department of Internal Medicine, University Hospital "Dr. Jose E. \\ Gonzalez", Autonomous University of Nuevo Leon, Monterrey, 64460, Mexico \\ ${ }^{8}$ Laboratorio Nacional para el Estudio y Aplicación de la Medicina Basada en Evidencia, Análisis \\ Crítico de la Información Científica y Farmacoeconomía, Universidad Autónoma de Nuevo León, \\ Monterrey, 66460, México \\ ${ }^{9}$ Women's Health Clinic, Division of General Internal Medicine, Mayo Clinic, Rochester, MN, \\ 55905, USA \\ ${ }^{10}$ Mayo Clinic Libraries, Rochester, MN, 55905, USA \\ ${ }^{11}$ Evidence-based Practice Center, Mayo Clinic, Rochester, MN, 55905, USA
}

\begin{abstract}
Purpose-Sex hormones play a role in bone density, cardiovascular health and wellbeing throughout reproductive lifespan. Women with primary ovarian insufficiency (POI) have lower estrogen levels requiring hormone therapy (HT) to manage symptoms and to protect against adverse long-term health outcomes. Yet, the effectiveness of HT in preventing adverse outcomes
\end{abstract}

Corresponding author: Dahima Cintron, PO Box 702 Barranquitas, PR 00794. Dahima.cintron1@ upr.edu, Phone: (787) 590-4551, ORCID: 0000-0003-4607-0234.

Authors contributed equally to this work.

Compliance with Ethical Standards

Conflict of Interest: SF is currently a consultant for Mithra Pharmaceuticals. All other authors declare no conflict of interest.

Ethical Approval: This article does not contain any studies with human participants performed by any of the authors. 
has not been systematically assessed. We summarize the evidence regarding effects of HT on bone and cardiovascular health in women with POI.

Methods-A comprehensive search of the electronic databases MEDLINE, EMBASE and Scopus was conducted by a medical reference librarian from database inception to January 2016. Randomized trials and observational cohort studies with an estrogen-based HT intervention in women with POI under the age of 40 were included. Reviewers worked independently and in duplicate to assess eligibility and risk of bias, and extract data of interest from each study.

Results-The search identified 1670 articles; 12 met inclusion criteria. Four randomized clinical trials and 8 cohort studies at high risk of bias enrolled 806 women with POI. The most common HT formulations were transdermal estradiol and oral conjugated equine estrogen combined with medroxyprogesterone acetate. Bone mineral density was the most frequent outcome, with 3 out of 8 studies showing HT associated increase benefits. Only 1 study reported effects on fractures or vasomotor symptoms and none on cardiovascular mortality. Results regarding lipid profiles were inconsistent.

Conclusions-Evidence supporting bone and cardiovascular benefits of HT in women with POI is limited by high risk of bias, reliance on surrogate outcomes and heterogeneity of trials regarding the formulation, dose, route of administration and regimen of HT. Further research addressing patient important outcomes such as fractures, stroke and cardiovascular mortality are crucial to optimize benefits of this therapy.

Registration-PROSPERO CRD 4201603616

\section{Keywords}

Primary ovarian insufficiency; primary ovarian failure; estrogen; hormone therapy; fracture; lipids; vasomotor symptoms; quality of life; adverse events

\section{Introduction}

Ovarian hormones are key regulators in bone, cardiovascular and general health throughout women's lives, from menarche to menopause when endogenous production declines [1]. This sequence may be interrupted prematurely by primary ovarian insufficiency (POI), where $1 \%$ of women present with either primary or secondary hypergonadotrophic hypogonadism [2]. Primary causes range from genetic to autoimmune; secondary causes include chemotherapy, radiotherapy, and bilateral oophorectomy [3]. Although the underlying etiology for hypogonadism in POI remains inconclusive in up to 70-90\% of cases, it may be due to a decreased number or increased rate of loss of ovarian follicles [4]. In contrast to the complete cessation of menstrual cycles observed in natural menopause occurring at the average age, POI may present with long-term intermittent and unpredictable ovarian function [5].

Women with POI have a two-fold increase in mortality associated with the increased incidence of cardiovascular disease, and higher incidence of osteoporosis [6]. Associated estrogen deficiency symptoms may also affect quality of life. These adverse outcomes have also been reported in young women with secondary POI due to bilateral oophorectomy [7], 
with outcome reports including overall acceleration of the aging process [8]. Although guidelines recommend continuous use of hormone therapy (HT) until the average age of menopause [2 9], studies show a mean duration of about one year [10].

The cardiovascular and bone benefits of HT in this population remain poorly defined; randomized controlled trials (RCT) are lacking, and evidence of plausible benefit has been extrapolated from studies conducted in women who reach menopause at the average age (51 years in the US) or in women with Turner syndrome [2]. Long-term health outcomes related to the use of HT in young women with POI may not be the same as for women who are menopausal at the average age. Discrepancies may also exist between women with POI from different etiologies.

This systematic review aims to summarize the body of evidence from randomized clinical trials and cohort studies regarding the effects of HT on bone health, cardiovascular risk, vasomotor symptoms, quality of life and adverse events in women with POI.

\section{Methods}

This review adheres to the Preferred Reporting Items for Systematic Reviews and Metaanalysis (PRISMA) Statement ${ }^{14}$ and was guided by a registered protocol (Prospero CRD 42016036166). Screening and extraction of articles were performed using online software (https://www.covidence.org/).

\section{Sources}

An experienced reference librarian (PJE) worked with principal investigator (DC) to develop a comprehensive search strategy. A combination of MeSH terms (Primary Ovarian Insufficiency, Turner syndrome) and text words (ovarian within 3 words of insufficiency, failure, and dysfunction) were selected to find original articles or abstracts, in any language, of women with hypergonadotropic hypogonadism. MEDLINE, EMBASE and Scopus were searched from each database inception to January 2016.

The full search strategy is detailed in APPENDIX 1. Data on patients with hypergonadotropic hypogonadism in women with Turner syndrome were summarized separately[11] and excluded from this systematic review. A post-hoc search was performed April 2017 in clinicaltrials.gov to evaluate for ongoing trials addressing the same population and intervention (APPENDIX 2).

\section{Study Selection}

Three reviewers (DC, PL, VS), working as independent pairs screened titles, abstracts and full text manuscripts for eligibility. There were no exclusion criteria based on language or publications status. After full-text screening, chance-adjusted agreement was quantified using the kappa statistic [12] and disagreements were resolved by consensus. Studies were screened for inclusion based on the following criteria:

- Design: randomized clinical trials and cohort studies (prospective, retrospective or pre-post treatment). 
- $\quad$ Population: women under age 40 , as cut-off to define premature age for amenorrhea[13]; that present with hypergonadotropic hypogonadism as evidenced by laboratory values of follicle stimulating hormone (FSH) levels greater than $25 \mathrm{mIU} / \mathrm{mL}$ [2] with either primary (idiopathic POI) or secondary (defined as chemotherapy, radiation and surgery) causes of hypergonadotropic hypogonadism.

- Intervention: HT interventions of either estrogen (primarily either oral or transdermal estradiol (o-E2 or t-E2) or oral conjugated equine estrogens [o$\mathrm{CEE}]$ ) alone or combined with progestogen. Regimens of progestogen could be either continuous or cyclical. We did not restrict studies based on type of estrogen, dose, route of administration or duration.

- $\quad$ Outcomes: bone health through frequency of fractures at any anatomical site and/or changes in BMD at lumbar spine, femur neck or total hip by dual energy $\mathrm{x}$-ray absorptiometry (DEXA). Cardiovascular health, through cardiovascular morbidity (stroke or myocardial infarction) and mortality and/or changes in metabolic lipid profile [total cholesterol, high density lipoprotein (HDL), low density lipoprotein (LDL) and triglycerides (TG)]. We included studies that used questionnaires to address quality of life, adverse effects and relief of vasomotor symptoms (hot flashes or night sweats).

\section{Data collection}

Data were extracted independently and in duplicate using a standardized data extraction form. Data collected included study details, population characteristics and HT description, including formulation, dose, route of administration, duration and regimen. Only data from eligible etiologies of POI (e.g. idiopathic, secondary to BMT, and surgery) are included in this report. Raw outcomes measures were extracted as change from baseline and at follow up. If data were missing, authors of included studies were contacted by email for an opportunity to send missing data in the next 8 weeks. Authors received a reminder email after two weeks.

\section{Risk of Bias}

The methodological quality of each study was assessed using the Cochrane Risk of Bias tool (CRB) (for RCTs) and the Newcastle-Ottawa Quality Assessment Scale (NOS) (for cohort studies). RCTs were determined to be at high risk of bias if there was either incomplete or selective outcome reporting. For studies with comparison groups that did not meet inclusion criteria, only the intervention arm (ie. pre-post design) was included without the comparator data. We did not derive a quantitative score from the NOS; rather, a judgment on the overall risk of bias of cohort studies was made based on the adequacy of cohort follow up and clarity of cohort description. 


\section{Results}

\section{Search Results}

Our search identified 1,666 reports, complemented by four hand-searched references, for a total of 1,670 titles and abstracts screened. FIGURE 1 demonstrates the study selection process. We reproducibly $(k=0.92)$ included 12 studies: five observational studies [14-18], three pre-post [19-21], and four RCTs [22-25], enrolling 806 participants of interest. The small number of studies and the heterogeneity of the studies in terms of included participants, interventions, comparators and outcomes (FIGURE 2) precluded statistical pooling. For ongoing clinical trials (APPENDIX 2), a total of 8 trials were registered: 3 completed, 2 not yet started, 1 withdrawn prior to enrollment, 1 with unknown status and a single study actively recruiting.

\section{Study Characteristics}

TABLE 1 describes included studies. Four [ 17212324$]$ of 12 studies were conducted in women with POI due to chemotherapy or bone marrow transplantation; six included women with idiopathic POI [14-16 1920 22], and two included women with more than one etiology of POI (mixed populations) [18 25]. The most common formulations of hormone therapy were t-E2 and o-CEE combined with medroxyprogesterone acetate (MPA); duration of therapy ranged from 6 to 120 months.

\section{Risk of Bias}

Four longitudinal cohort studies were rated at high risk of bias [ $\left.\begin{array}{lllll}14 & 16 & 18 & 20\end{array}\right]$ and three at low risk of bias (TABLE 2) [15 19 21]. All RCTs were rated at high risk of bias (TABLE 3). Common parameters that resulted in ratings of high risk of bias included inadequate followup in observational studies and selective outcome reporting in RCTs.

\section{Outcomes}

Cardiovascular Health-Outcomes of included study are reported in FIGURE 2. Two of six studies in women with idiopathic POI reported changes in lipid profile after o-CEE + MPA interventions [15 19]. A prospective cohort evaluating changes in lipid profile in a group of 18 female patients over a 6-month period reported a significant decrease in LDL and increase in TG as compared to baseline [15]. A pre-post study of 9 female patients showed no significant changes in total cholesterol, LDL, HDL or triglycerides after 9 months [19]. No conclusions could be drawn from an RCT that included 2 participants of interest with POI secondary to chemotherapy BMT receiving o-CEE+MP, as authors reported changes based on HRT formulation and not by etiology of hypogonadism [24]. A two-year study which included a subset of patients with POI of eligible etiologies reported that between 10 women with POI treated with o-CEE plus a progestogen versus 5 women with POI who were untreated, there were no changes in serum lipid levels. Results were not stratified and included women with anorexia nervosa, Turner syndrome and hypogonadotropic hypogonadism [18].

Bone Health-Five of the 6 included studies evaluating patients with idiopathic POI [14 16192022 ] reported bone health outcomes by presenting data as bone mineral density 
$\left(\mathrm{g} / \mathrm{cm}^{2}\right)$, Z-score and/or T-score. One RCT that evaluated BMD after estradiol/progestogen replacement compared to either estradiol/progestogen/testosterone or women without POI, found a significant increase in total spine and femur neck bone mineral density when compared to their baseline values. At baseline, the HT treated group had a lower BMD than controls ( $\left.0.77 \mathrm{vs} 0.81 \mathrm{~g} / \mathrm{cm}^{2}, \mathrm{p}=0.001\right)$ a difference that disappeared at the end of the study ( $0.80 \mathrm{~g} / \mathrm{cm}^{2}$ in both groups) [22]. A study comparing effects of HT on 28 women with POI to premenopausal, menstruating women found that at baseline, BMD was evidently lower than in age-matched premenopausal controls, and after HT, it increased at a rate faster than that of women without HT [14]. A pre-post study following women every 4 months for one year, found that in the 9 patients given o-CEE, a significant increase was observed in total hip BMD [19]. In contrast, a cohort study that evaluated BMD at total spine and femur neck in 72 women receiving either o-CEE, o-E2 or o-ethinyl estradiol (o-EE) over the course of 8 years identified no statistically significant change in BMD with no distinction between the various HT formulations [20].

A single RCT reported fractures across 60 female patients with POI secondary to chemotherapy for BMT randomized to receive either: calcium + vitamin D, calcium + vitamin D + estradiol + dihydroprogesterone (intervention), risendroate, or zolendroic acid for an average follow-up of 12 months. For this systematic review, the intervention group was compared to the group receiving only calcium + vitamin D. After 12 months, 2/15 patients receiving calcium and vitamin $\mathrm{D}$, and $1 / 15$ patients receiving the intervention developed vertebral fractures [23]. A pre-post study evaluated 13 patients with POI secondary to BMT for chemotherapy receiving ( $t-E 2$ or o-CEE) + MPA found an increase in bone mineral content in all participants after 12 months of HRT [21].

One RCT directly comparing o-EE and t-E2 showed a significant benefit of t-E2 over o-EE on bone mass in a subset of patients with POI of eligible etiologies. Over 12 months of intervention t-E2 showed a z-score increase of 0.17 compared to a 0.07 increase in o-EEtreated women. Results are not stratified by etiology of POI, with significant differences in baseline characteristics between women who completed versus those who withdrew from the study [25].

\section{Quality of Life Related Issues}

Only a single study of those included in this review evaluated quality of life related issues, such as vasomotor symptoms, and did so in a population of women with POI after BMT. This prospective cohort evaluated the percent of women with POI, after total body irradiation due to bone marrow transplant, presenting with vasomotor symptoms at baseline and at 6 months utilizing either o-CEE or t-E2 plus MPA. At baseline a total of 78\% of women (58 out of 74 ) reported vasomotor symptoms. After a loss to follow up of close to $50 \%$ at 6 months, $91 \%$ of remaining participants reported improvement of their VMS (31 out of 34) [17]. This study compared systemic treatment (transdermal and oral) with local treatment; data comparing response of vasomotor symptoms after transdermal or oral HRT was not evaluated in this study. 


\section{Discussion}

Summary of findings-In the present systematic review, we evaluated 12 studies which assessed the effects of HT in women with POI. Among included studies none evaluated patient important outcomes such as cardiovascular events, mortality, or quality of life. Of the 12 studies included, 8 were found to have a high risk of bias. The current review shows evidence has largely focused on surrogate outcomes. This poses a challenge to clinical decision making as the relationship between surrogate markers and patient important outcomes in women with POI remains uncertain.

Limitations and Strengths-The present systematic review was limited by the small number of studies included for qualitative synthesis. Inability to conduct meta-analysis and provide estimates of effect is another limitation. Conclusions about the effects of HT were often limited by incomplete and short length of follow-up. A major concern that stems from this synthesis of evidence is that studies are pooling data from women who present with hypogonadism due to different etiologies. This results in evidence that is indirectly applied to specific subgroups of women with POI and limits the applicability of the results to individual patients. Additionally, the formulations, doses, and routes of administration of estrogen, and the concomitant use of a progestogen in women with a uterus vary or are not clearly defined in many of the studies, making it difficult to generalize conclusions. Unfortunately, only one study included evaluated HRT effect on VMS in patients with POI and did so in a subset of patients with POI secondary to chemotherapy for BMT. There is limited data available regarding quality of life related issues in patients with POI of diverse etiologies.

This review is strengthened by the comprehensive search strategy employed. It addresses longitudinal studies of women with POI including RCTs and observational longitudinal studies. The selection criteria include a study population of women with either idiopathic POI or secondary POI, highlighting the need for subgroup analyses based on the etiology of POI to limit confounding by these factors. Although the evidence that women with POI are at significantly increased risk for fractures, coronary heart disease and cardiovascular mortality is clear [2], it is unclear if HT reduces these risks. There is no standard recommendation for dosing or target serum hormone levels to be achieved with HT, as data regarding the doses or serum levels needed to protect against long-term adverse cardiovascular outcomes is lacking [7]. Recommendations for HT in women with POI are based primarily on extrapolation of outcomes in women experiencing menopause at the average age, women with Turner syndrome [2], or in women who have undergone bilateral oophorectomy before the age of 45 years [26 27]. Yet large randomized trials involving women with various subtypes of POI, including but not limited to early bilateral oophorectomy, are needed, considering the formulation, dose, and route of administration of hormone therapy used.

The studies included in this systematic review did not report outcomes related to risks of HRT. In addition, studies with small sample sizes are unlikely to adequately assess the risk of rare side effects and harm; large studies, following patients over long periods of time are needed for a better assessment of these risks [28]. Unfortunately, adverse effects of HRT 
reported in studies with post-menopausal women have been, perhaps, erroneously translated to younger women with POI; the true risk of harm in this population is yet to be determined empirically[2 2029$]$.

Comparison with previous literature-We present, to our knowledge, the first systematic review summarizing the effects of HT in women with POI. A previous systematic review and meta-analysis including only women with Turner syndrome showed that HT was associated with increased BMD and HDL cholesterol when compared to baseline values, with minimal reporting of patient important outcomes such as fractures, cardiovascular mortality or vasomotor symptoms [11]. Other etiologies of POI, however, are increasing in prevalence. With advances in treatments, more women survive childhood cancers, and the number of patients living with secondary POI is expected to rise. Although studies in women who are menopausal at the average age have shown improvement in VMS and some menopause-related sleep disturbances with HT [30], it is unclear if these findings can be extrapolated to women with POI. In addition, it remains unclear if the doses of estrogen used in these studies were sufficient to approximate physiologic levels found in premenopausal menstruating women as recommended by some experts [7 29].

Implications for research and practice-Future prospective studies with protection against risk for bias are urgently needed in this field. Important factors that might modify the treatment response have not been compared in subgroup analyses. Among these, the most important are the formulation, dose and route of administration of estrogen-based HT and the etiology for POI. Additionally, the impact of other ovarian hormones potentially affected by POI (e.g., testosterone and progesterone) on long-term health outcomes is also unclear. Women with POI discontinue treatment after an average of one year, thus understanding barriers to treatment is critical. Importantly, the results of HT trials in women who are menopausal at the average age cannot be extrapolated to women experiencing early or premature menopause. Current guidelines recommend the use of HT at least until the average age of menopause (age 51 years), with consideration of longer-term therapy based on symptom management [9 31]. Additionally, the emotional sequelae of a POI diagnosis can be difficult and needs to be addressed in clinical management [5].

\section{Conclusion}

The current body of evidence of HT effects on bone and cardiovascular health in women with POI is limited to surrogate outcomes, with studies demonstrating variable degrees of risk of bias. Given the risk of potential adverse long-term health consequences in women with premature menopause who do not receive HT and the lack of demonstrable harm (except in the presence of a hormone-dependent cancer), the use of HT is currently encouraged at least until the natural age of menopause (51 years). Although high quality direct evidence is lacking, indirect evidence (for example, increased mortality in women who underwent bilateral oophorectomy before age 45 years) supports the use of estrogen doses that yield physiologic levels in premenopausal women. Clinicians should be aware of the remaining knowledge gaps, and thoroughly inform patients of the benefit versus risk ratio of HT use. Informed, shared decisions should be fostered, with strong emphasis on the potential benefits of HT replacement. Further research addressing fractures, cardiovascular 
outcomes including stroke and myocardial infarction, and vasomotor symptoms is crucial to determine and optimize the benefits of HT for this population of young women.

\section{Acknowledgments}

Funding: DC and GSB were supported by the CTSA Grant Number TL1 TR000137 from the National Center for Advancing Translational Science (NCATS), a component of the National Institutes of Health (NIH). GSB was also supported by grant number 3R01HL131535-01S1 from the National Heart Lung and Blood Institute (NHLBI). Its contents are solely the responsibility of the authors and do not necessarily represent the official view of the NIH.

\section{References}

1. De Vos M, Devroey P, Fauser BC. Primary ovarian insufficiency. Lancet. 2010; 376(9744):911-21. [published Online First: Epub Date]|. DOI: 10.1016/s0140-6736(10)60355-8 [PubMed: 20708256]

2. Webber L, Davies M, Anderson R, et al. ESHRE Guideline: management of women with premature ovarian insufficiency. Hum Reprod. 2016; 31(5):926-37. [published Online First: Epub Date]|. DOI: 10.1093/humrep/dew027 [PubMed: 27008889]

3. Welt CK. Primary ovarian insufficiency: a more accurate term for premature ovarian failure. Clin Endocrinol (Oxf). 2008; 68(4):499-509. [published Online First: Epub Date]|. DOI: 10.1111/j. 1365-2265.2007.03073.x [PubMed: 17970776]

4. Vujovic S. Aetiology of premature ovarian failure. Menopause Int. 2009; 15(2):72-5. [published Online First: Epub Date]|. DOI: 10.1258/mi.2009.009020 [PubMed: 19465673]

5. Nelson LM, Covington SN, Rebar RW. An update: spontaneous premature ovarian failure is not an early menopause. Fertil Steril. 2005; 83(5):1327-32. [published Online First: Epub Date]|. DOI: 10.1016/j.fertnstert.2004.11.059 [PubMed: 15866564]

6. Snowdon DA, Kane RL, Beeson WL, et al. Is early natural menopause a biologic marker of health and aging? Am J Public Health. 1989; 79(6):709-14. [PubMed: 2729468]

7. Faubion SS, Kuhle CL, Shuster LT, Rocca WA. Long-term health consequences of premature or early menopause and considerations for management. Climacteric. 2015; 18(4):483-91. [published Online First: Epub Date]|. DOI: 10.3109/13697137.2015.1020484 [PubMed: 25845383]

8. Rocca WA, Gazzuola Rocca L, Smith CY, et al. Bilateral Oophorectomy and Accelerated Aging: Cause or Effect? J Gerontol A Biol Sci Med Sci. 2017; doi: 10.1093/gerona/glx026[published Online First: Epub Date]|

9. The 2012 hormone therapy position statement of: The North American Menopause Society. Menopause. 2012; 19(3):257-71. [published Online First: Epub Date]|. DOI: 10.1097/gme. 0b013e31824b970a [PubMed: 22367731]

10. Mittal M, Savvas M, Narvekar N, Panay N, Hamoda H. A cross-sectional national questionnaire survey assessing the clinical attitudes of members of the British Menopause Society to the management of women with premature ovarian insufficiency. Post Reprod Health. 2014; 20(3):907. [published Online First: Epub Date]|. DOI: 10.1177/2053369114540883 [PubMed: 24973048]

11. Cintron D, Rodriguez-Gutierrez R, Serrano V, Latortue-Albino P, Erwin PJ, Murad MH. Effect of estrogen replacement therapy on bone and cardiovascular outcomes in women with turner syndrome: a systematic review and meta-analysis. Endocrine. 2016; 55(2):366-75. [published Online First: Epub Date]|. DOI: 10.1007/s12020-016-1046-y [PubMed: 27473099]

12. McGinn T, Wyer PC, Newman TB, Keitz S, Leipzig R, For GG. Tips for learners of evidencebased medicine: 3. Measures of observer variability (kappa statistic). Cmaj. 2004; 171(11):1369_ 73. [published Online First: Epub Date]|. DOI: 10.1503/cmaj.1031981 [PubMed: 15557592]

13. Pitkin J, Rees MC, Gray S, et al. Management of premature menopause. Menopause Int. 2007; 13(1):44-5. [published Online First: Epub Date]|. DOI: 10.1258/175404507780456719 [PubMed: 17448268]

14. Metka M, Holzer G, Heytmanek G, Huber J. Hypergonadotropic hypogonadic amenorrhea (World Health Organization III) and osteoporosis. Fertility and sterility. 1992; 57(1):37-41. [PubMed: 1730328] 
15. Kalantaridou SN, Vanderhoof VH, Calis KA, Corrigan EC, Troendle JF, Nelson LM. Sexual function in young women with spontaneous $46, \mathrm{XX}$ primary ovarian insufficiency. Fertility and sterility. 2008; 90(5):1805-11. [published Online First: Epub Date]|. DOI: 10.1016/j.fertnstert. 2007.08.040 [PubMed: 17961560]

16. Chatterjee R, Katz M, Bajoria R. Use of hormone replacement therapy for correction of high turnover bone disease in hypogonadal $\beta$-thalassemia major patients presenting with osteoporosis: Comparison with idiopathic premature ovarian failure. Hemoglobin. 2011; 35(5-6):653-58. [published Online First: Epub Date]|. DOI: 10.3109/03630269.2011.630120 [PubMed: 22074125]

17. Spinelli S, Chiodi S, Bacigalupo A, et al. Ovarian recovery after total body irradiation and allogeneic bone marrow transplantation: long-term follow up of 79 females. Bone Marrow Transplant. 1994; 14(3):373-80. [PubMed: 7994257]

18. Emans SJ, Grace E, Hoffer FA, Gundberg C, Ravnikar V, Woods ER. Estrogen deficiency in adolescents and young adults: impact on bone mineral content and effects of estrogen replacement therapy. Obstetrics \& Gynecology. 1990; 76(4):585-92. [PubMed: 2170885]

19. Papagianni V, Deligeoroglou E, Makrakis E, Botsis D, Creatsas G. Response to hormonal treatment of young females with primary or very premature ovarian failure. Gynecological Endocrinology. 2011; 27(5):291-99. [published Online First: Epub Date]|. DOI: 10.3109/09513591003632274 [PubMed: 21381875]

20. Benetti-Pinto CL, Ferreira VB, Yela DA. Long-term follow-up of bone density in women with primary ovarian insufficiency. Menopause. 2015; 22(9):946-9. doi: http://dx.doi.org/10.1097/ GME.0000000000000445. [published Online First: Epub Date]|. [PubMed: 25756693]

21. Castelo-Branco C, Rovira M, Pons F, et al. The effect of hormone replacement therapy on bone mass in patients with ovarian failure due to bone marrow transplantation. Maturitas. 1996; 23(3): 307-12. [PubMed: 8794425]

22. Popat VB, Calis KA, Kalantaridou SN, et al. Bone Mineral Density in Young Women With Primary Ovarian Insufficiency: Results of a Three-Year Randomized Controlled Trial of Physiological Transdermal Estradiol and Testosterone Replacement. Journal of Clinical Endocrinology \& Metabolism. 2014; 99(9):3418-26. [published Online First: Epub Date]|. DOI: 10.1210/jc.2013-4145 [PubMed: 24905063]

23. Tauchmanovà L, De Simone G, Musella T, et al. Effects of various antireabsorptive treatments on bone mineral density in hypogonadal young women after allogeneic stem cell transplantation. Bone Marrow Transplantation. 2006; 37(1):81-88. [published Online First: Epub Date]|. DOI: 10.1038/sj.bmt.1705196 [PubMed: 16247420]

24. Shah S, Forghani N, Durham E, Neely EK. A randomized trial of transdermal and oral estrogen therapy in adolescent girls with hypogonadism. International Journal of Pediatric Endocrinology. 2014; 2014(1) [published Online First: Epub Date]|. doi: 10.1186/1687-9856-2014-12

25. Crofton PM, Evans N, Bath LE, et al. Physiological versus standard sex steroid replacement in young women with premature ovarian failure: Effects on bone mass acquisition and turnover. Clinical Endocrinology. 2010; 73(6):707-14. [published Online First: Epub Date]|. DOI: 10.1111/j.1365-2265.2010.03868.x [PubMed: 20738314]

26. Shuster LT, Gostout BS, Grossardt BR, Rocca WA. Prophylactic oophorectomy in premenopausal women and long-term health. Menopause Int. 2008; 14(3):111-6. [published Online First: Epub Date]|. DOI: 10.1258/mi.2008.008016 [PubMed: 18714076]

27. Parker WH, Feskanich D, Broder MS, et al. Long-term mortality associated with oophorectomy compared with ovarian conservation in the nurses' health study. Obstet Gynecol. 2013; 121(4): 709-16. [published Online First: Epub Date]|. DOI: 10.1097/AOG.0b013e3182864350 [PubMed: 23635669]

28. Guyatt, G., Rennie, D., Meade, MO., Cook, DJ. Users' Guides to the Medical Literature: A Manual for Evidence-Based Clinical Practice. New York: MCGRAW-HILL EDUCATION - EUROPE; 2015.

29. Nelson LM. Clinical practice. Primary ovarian insufficiency. N Engl J Med. 2009; 360(6):606-14. [published Online First: Epub Date]|. DOI: 10.1056/NEJMcp0808697 [PubMed: 19196677]

30. Cintron D, Lipford M, Larrea-Mantilla L, et al. Efficacy of menopausal hormone therapy on sleep quality: systematic review and meta-analysis. Endocrine. 2016; doi: 10.1007/ s12020-016-1072-9[published Online First: Epub Date]| 
31. de Villiers TJ, Hall JE, Pinkerton JV, et al. Revised Global Consensus Statement on Menopausal Hormone Therapy. Climacteric. 2016; 19(4):313-5. [published Online First: Epub Date]|. DOI: 10.1080/13697137.2016.1196047 [PubMed: 27322027] 

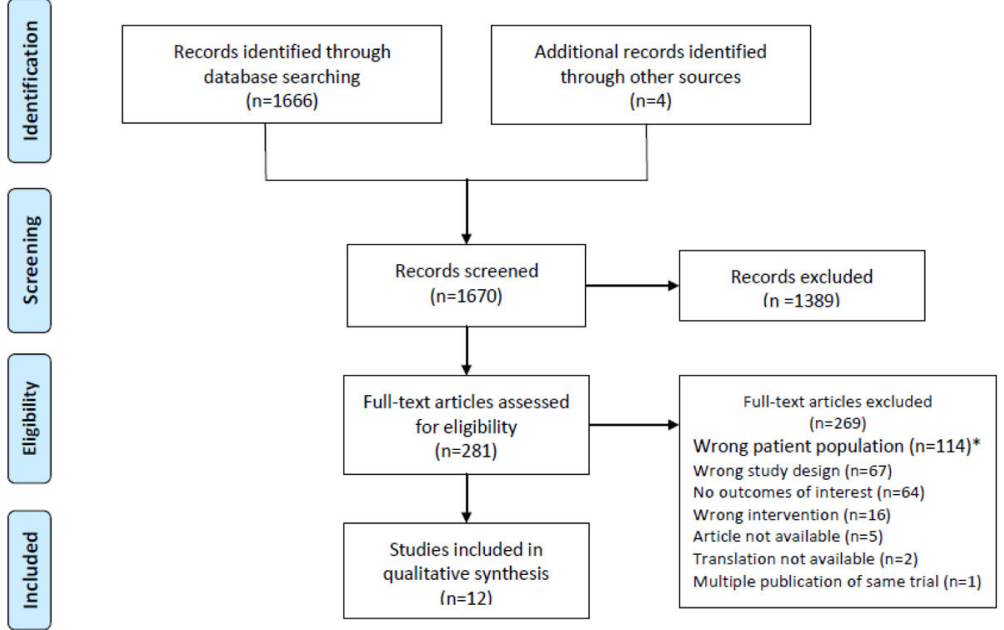

FIGURE 1.

* A total of 21 studies had women with Turner Syndrome; these were assessed in a separate systematic review, adjoint with 4 included studies looking at women with Premature Ovarian Insufficiency and Turner Syndrome together. 


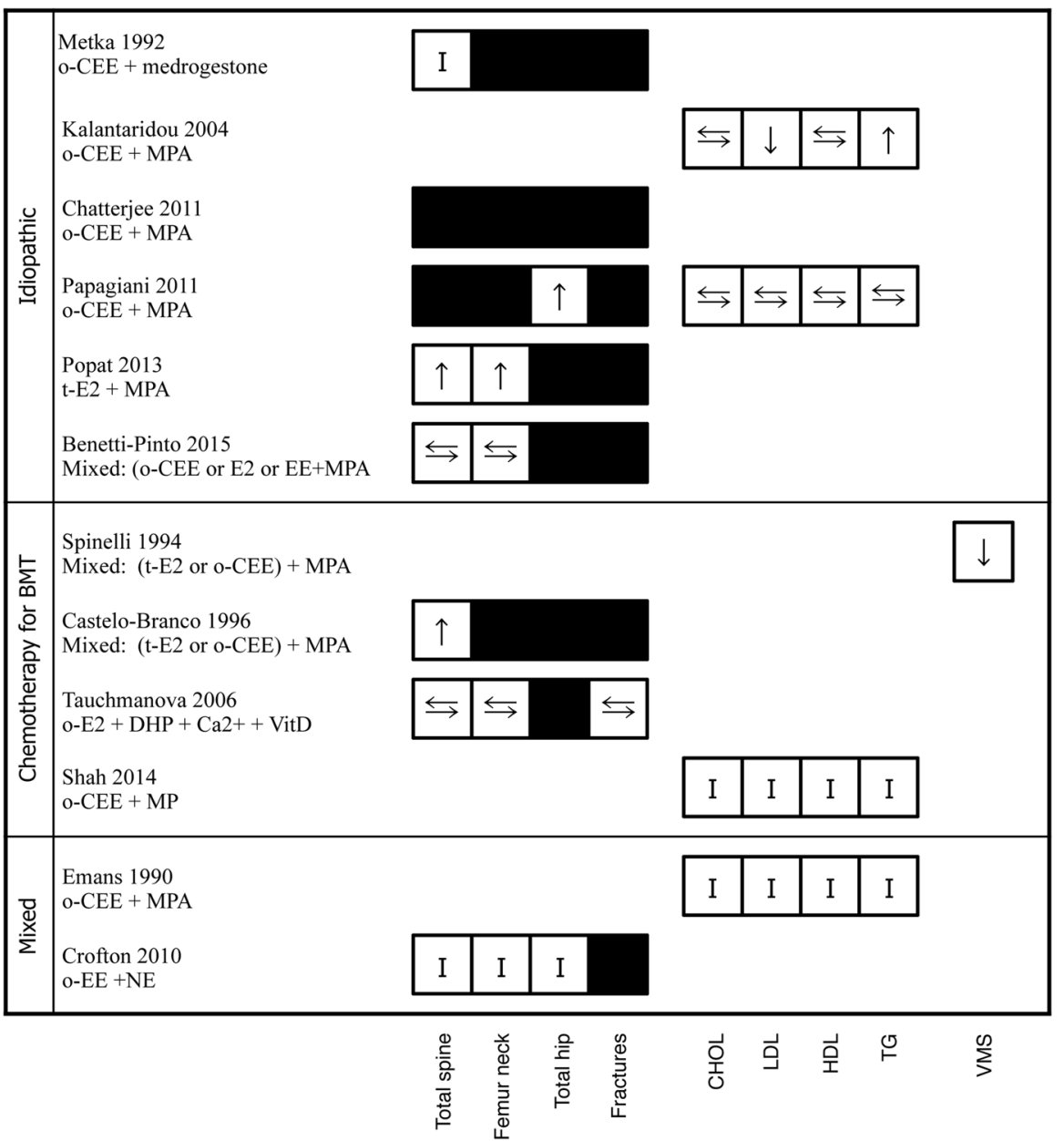

FIGURE 2.

Bone, lipid and vasomotor symptoms results by study divided by premature ovarian insufficiency etiology. Abbreviations: $\uparrow$, significant increase; $\downarrow$ significant decrease; $\leftrightarrows$, no significant change; I, Inconclusive; results for population of interest were not reported independently. Parameter was not studied or reported. 


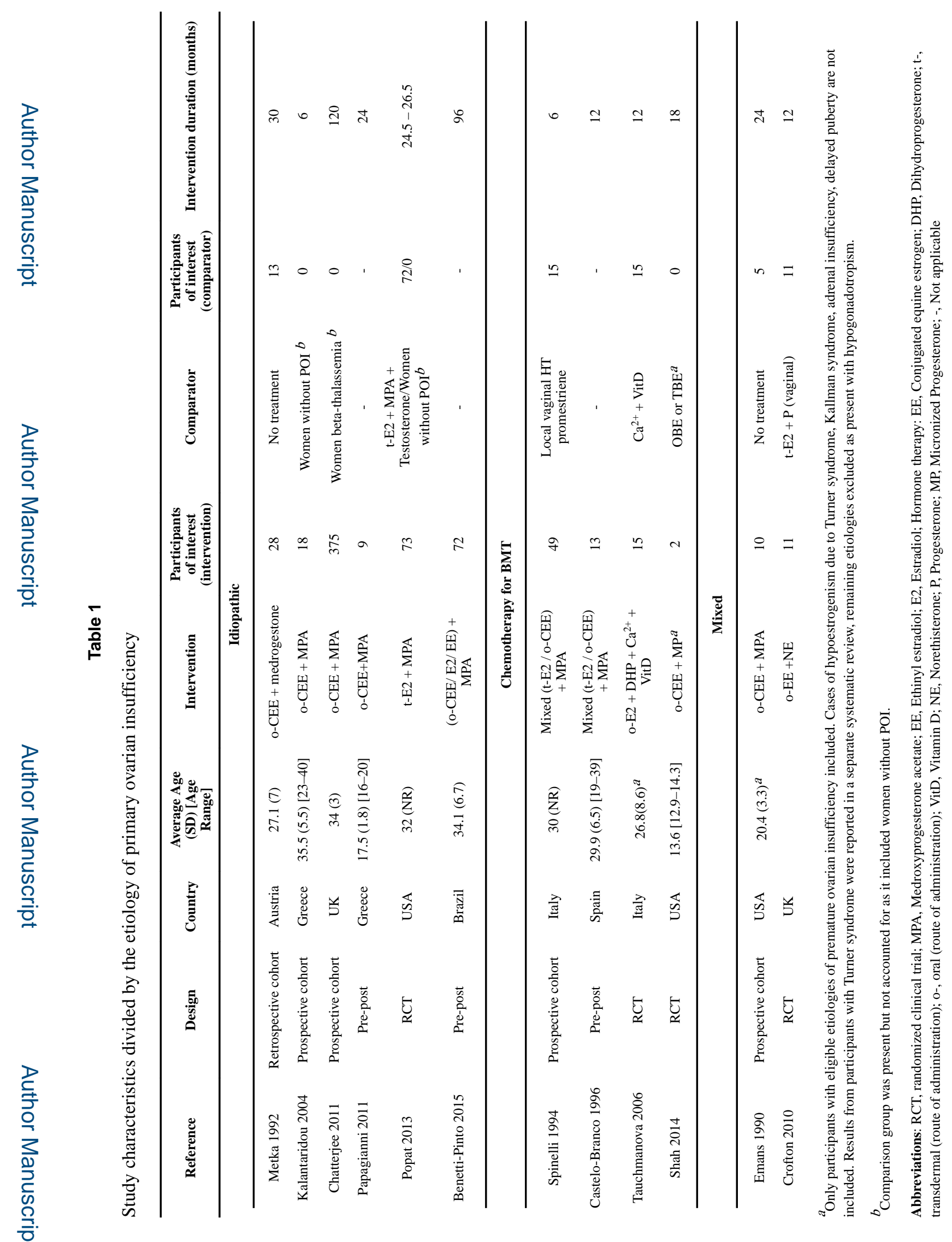

Endocrine. Author manuscript; available in PMC 2018 December 01. 


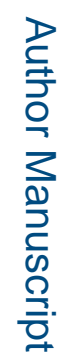

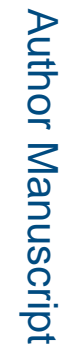

ह

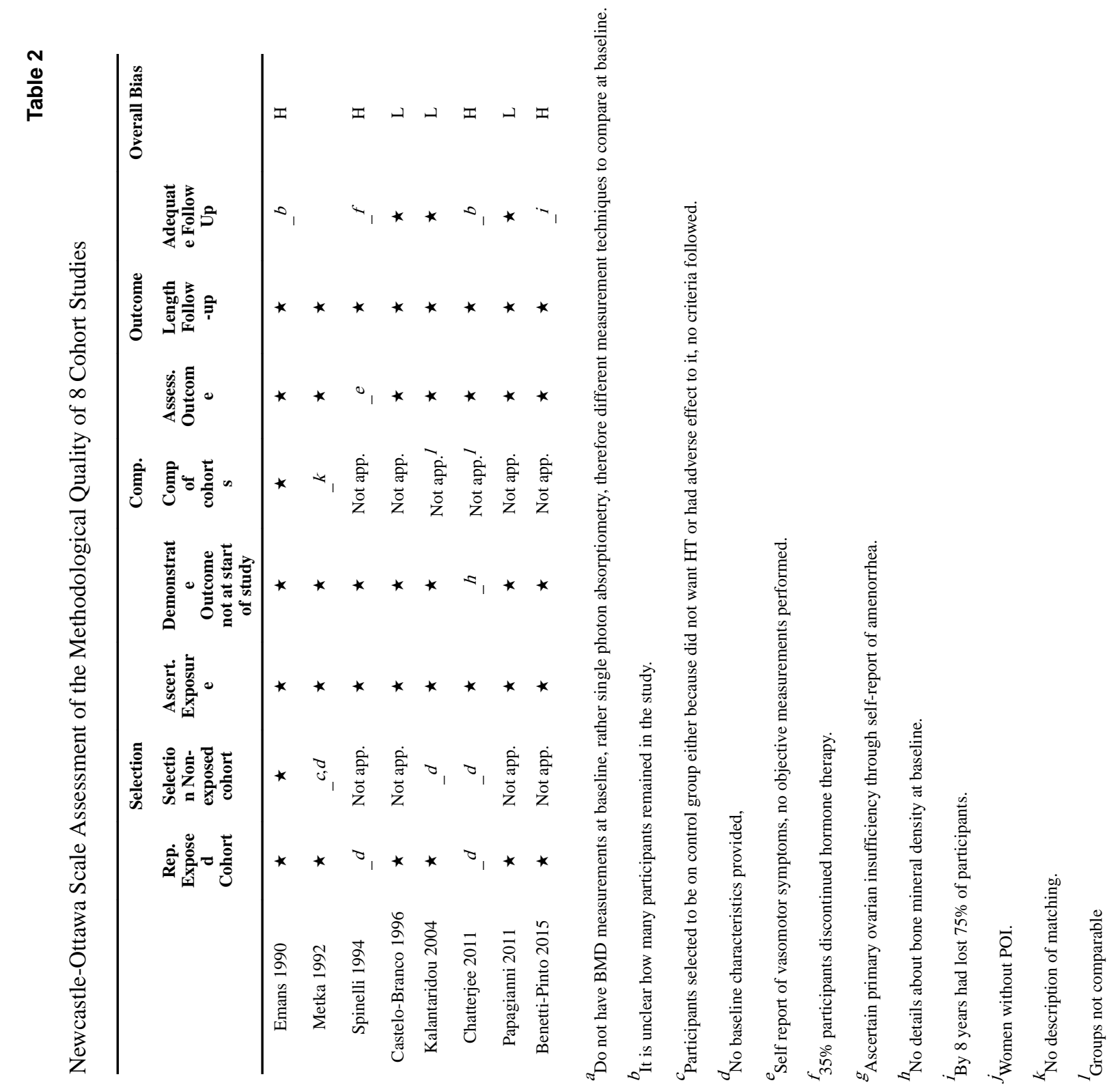



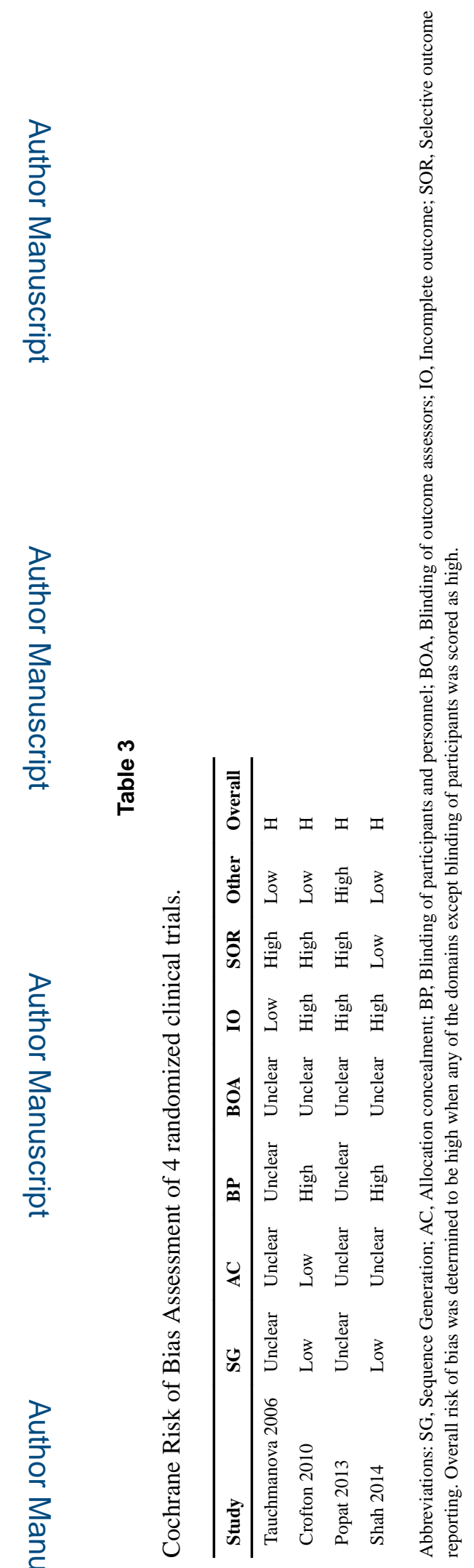

ᄅ⿳亠口冋.
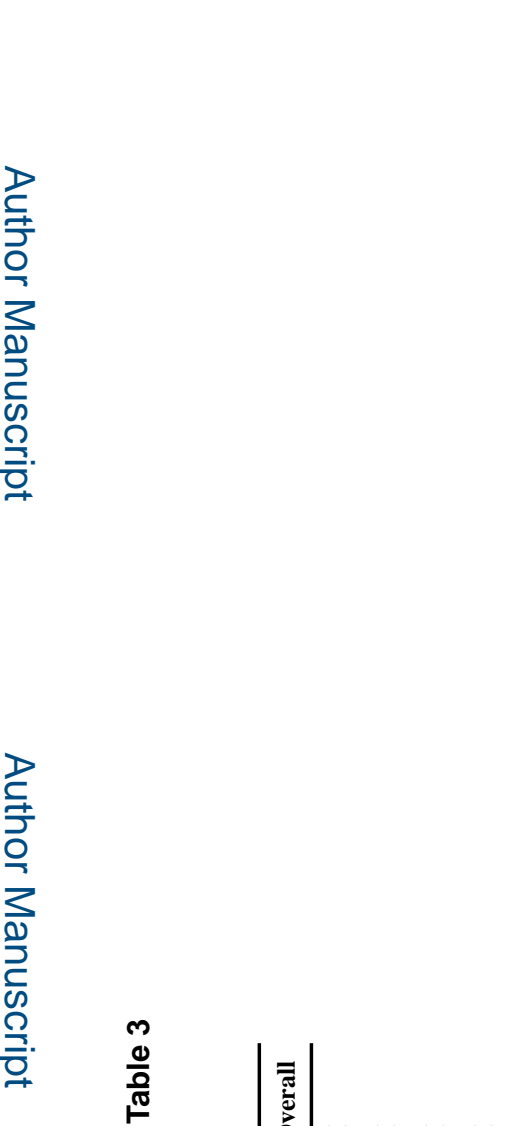

Endocrine. Author manuscript; available in PMC 2018 December 01. 